\section{Resolution of human ribosomal DNA occurs in anaphase, dependent on tankyrase 1 , condensin II, and topoisomerase IIa}

\author{
Zharko Daniloski, ${ }^{1}$ Kamlesh K. Bisht, ${ }^{1,3}$ \\ Brian McStay, ${ }^{2}$ and Susan Smith ${ }^{1}$ \\ ${ }^{1}$ Department of Pathology, Kimmel Center for Biology and \\ Medicine at the Skirball Institute, New York University School \\ of Medicine, New York, New York 10016, USA; ${ }^{2}$ Centre for \\ Chromosome Biology, School of Natural Sciences, National \\ University of Ireland Galway, Ireland
}

Formation of individualized sister chromatids is essential for their accurate segregation. In budding yeast, while most of the genome segregates at the metaphase to anaphase transition, resolution of the ribosomal DNA (rDNA) repeats is delayed. The timing and mechanism in human cells is unknown. Here we show that resolution of human rDNA occurs in anaphase after the bulk of the genome, dependent on tankyrase 1 , condensin II, and topoisomerase II $\alpha$. Defective resolution leads to rDNA bridges, rDNA damage, and aneuploidy of an rDNA-containing acrocentric chromosome. Thus, temporal regulation of rDNA segregation is conserved between yeast and man and is essential for genome integrity.

Supplemental material is available for this article.

Received October 22, 2018; revised version accepted January 7, 2019.

Resolution of sister chromatids in mitosis requires that cohesin be removed, sister chromatids be condensed, and intersister linkages be untangled (Shintomi and Hirano 2010; Kschonsak and Haering 2015). In budding yeast, sister chromatids are cohered along their arms and centromeres until metaphase (Guacci et al. 1997; Michaelis et al. 1997). At anaphase onset, proteolytic cleavage of cohesin rings triggers sister chromatid separation (Uhlmann et al. 1999, 2000). However, separation is delayed for some specialized repetitive sequences (telomeres and ribosomal DNA [rDNA]) until late anaphase (D'Amours et al. 2004; Sullivan et al. 2004; Torres-Rosell et al. 2004; Wang et al. 2004). In human cells (unlike in yeast), resolution of telomeres occurs at the same time as arms and takes place in G2/prophase prior to centromere resolution (Waizenegger et al. 2000; Ofir et al. 2002; Canudas and Smith 2009; Bisht et al. 2013). Centromeres then resolve at anaphase onset upon cohesin cleavage (Waizenegger et al. 2000). Despite occurring at the same time as chromosome arms (in G2/prophase), resolution of human telo-

[Keywords: condensin; rDNA; tankyrase; topoisomerase IIa] ${ }^{3}$ Present address: Celgene Corp., Summit, New Jersey 07901, USA. Corresponding author: susan.smith@med.nyu.edu

Article published online ahead of print. Article and publication date are online at http://www.genesdev.org/cgi/doi/10.1101/gad.321836.118. meres (but not arms) requires the poly(ADP-ribose) polymerase tankyrase 1 (Smith et al. 1998). In the absence of tankyrase 1, arms and centromeres resolve normally, but telomeres remain cohered (Dynek and Smith 2004). Cells delay briefly in anaphase, but, ultimately, sister chromatids separate (Kim and Smith 2014). The timing for resolution of human rDNA, which (like telomeres) comprise a highly repetitive element in the genome, is not known. In human cells, a 13-kb pre-rRNA is transcribed by RNA polymerase I from a 43-kb gene (Gonzalez and Sylvester 1995) found in repetitive clusters on the short (p) arms of the five acrocentric chromosomes (Henderson et al. 1972). The rDNA genes are highly transcribed in nucleoli throughout interphase, until late prophase when transcription is repressed and nuclear envelope breakdown initiated (McStay 2016; Nemeth and Grummt 2018). How these specialized sequences resolve and segregate and the impact of aberrant resolution on genome stability has not been determined. Here we elucidate the mechanisms for accurate segregation of the human rDNA repeat arrays in mitosis.

\section{Results and Discussion}

To measure the resolution status of rDNA in mitosis, HeLa cells were isolated by mitotic shake-off from asynchronous cultures, (which yields mostly prophase cells), fixed immediately to preserve cohesion, and analyzed by fluorescent in situ hybridization (FISH) using a probe against the 43-kb rDNA gene that detects the arrays on the five acrocentric chromosomes $(13,14,15,21$, and 22) (Fig. 1A; Henderson et al. 1972). The number of rDNA repeats on each chromosome can vary greatly from one repeat $(43 \mathrm{~kb})$ to $>140$ repeats $(>6 \mathrm{Mb}$ ) (Stults et al. 2008). As shown in Figure 1B, we observed $\sim 10$ loci of varying size; most appeared as singlets (unresolved). Dual FISH with an acrocentric centromere 15 probe (trisomic in HeLa cells) revealed three single loci. Thus, like centromeres (but unlike arms and telomeres) rDNA remains unresolved in mitosis (Fig. 1B,C).

To determine whether the unresolved status was a property of the rDNA itself or a feature of the acrocentric parm, we performed FISH analysis using probes against junctional sequences that flank the rDNA arrays: the proximal junction (PJ) and the distal junction (DJ) (see Fig. 1A; Floutsakou et al. 2013). The DJ and PJ can be detected as 10 or more singlets in interphase cells (Supplemental Fig. S1A,B). In FISH analysis of mitotic cells, the majority of DJ signals appeared as doublets (resolved), in contrast to the rDNA singlets, indicating that sequences flanking the rDNA on the telomere side are resolved in mitosis (Fig. 1D,E). The same pattern was observed in normal BJ fibroblasts (Supplemental Fig. S1C,D). The PJ probe appeared similar to the DJ in mitotic cells; the majority of signals appeared as doublets (resolved) (Fig. 1F,G), indicating that the sequences flanking the rDNA on the

(C) 2019 Daniloski et al. This article is distributed exclusively by Cold Spring Harbor Laboratory Press for the first six months after the full-issue publication date (see http://genesdev.cshlp.org/site/misc/terms.xhtml). After six months, it is available under a Creative Commons License (Attribution-NonCommercial 4.0 International), as described at http://creativecommons.org/licenses/by-nc/4.0/. 
A

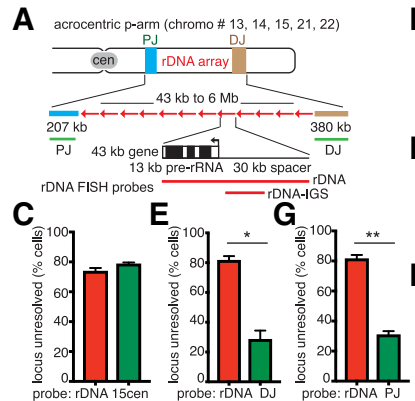

H

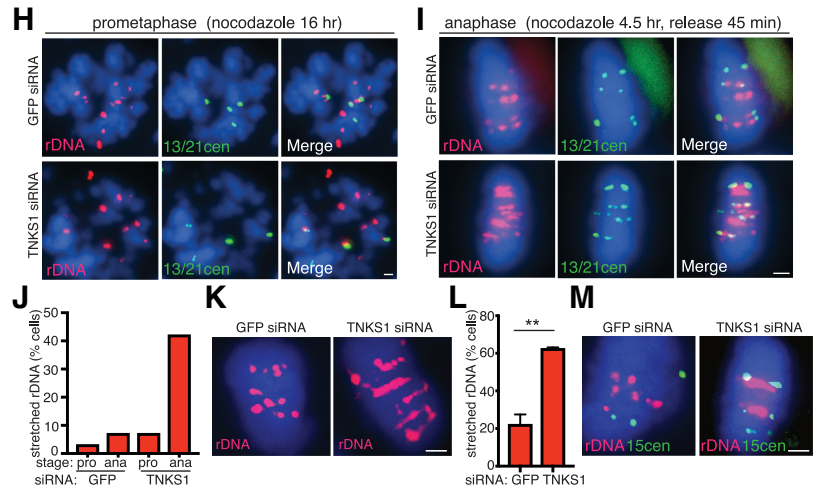

Figure 1. rDNA resolution is delayed until anaphase and depends on tankyrase 1. (A) Schematic diagram of the rDNA arrays on the p-arms of the five acrocentric human chromosomes. (PJ) Proximal junction; (DJ) distal junction. The repeating unit is a 43-kb gene comprised of pre-rRNA and untranscribed IGS (intergenic spacer). (B-G) rDNA is not resolved in prophase. FISH analysis of mitotic cells isolated by shake-off from asynchronously growing HeLa cells and probed for rDNA (red) $(B, D, F)$ and 15 cen (green) $(B), \mathrm{DJ}$ (green) $(D)$, or PJ (green) $(F)$. $(C, E, G)$ Quantification of results in $B, D$, and $F$, respectively. $\mathrm{Mi}$ totic cells were scored as having rDNA, DJ, or PJ unresolved if $\geq 50 \%$ of their loci appeared as singlets. Average of two independent experiments \pm SEM. $(C) n=54-83$ cells each. $(E) n=32$ cells each. $(G) n=50$ 51 cells each. $\left({ }^{*}\right) P \leq 0.05,\left({ }^{*}\right) P \leq 0.01$, Student's unpaired $t$-test. $(H-J)$ rDNA is resolved late in anaphase, dependent on tankyrase 1. Following a 48-h transfection with GFP or TNKS1 siRNA, HeLa cells were arrested in prometaphase by a 16-h nocodazole treatment $(H)$ or in anaphase by a 4.5 -h nocodazole treatment followed by a $45-\mathrm{min}$ release $(I)$, isolated by mitotic shake-off, and analyzed by FISH using rDNA (red) and 13/21 cen (green) probes. ( $J$ ) Quantification of results in $H$ and $I$. (pro) Prometaphase; (ana) anaphase. Mitotic cells with three or more stretched rDNA lines were scored as having stretched rDNA. For anaphase, only cells with separated centromeres were scored. $n=31-51$ cells. $(K-M)$ FISH analysis of rDNA stretching in mitotic cells isolated by shake-off from asynchronously growing $\mathrm{HeLa}$ cells following a 48-h transfection with GFP or TNKS1 siRNA and probed for rDNA (red) $(K-M)$ and 15 cen (green) $(M)$. Deconvolved images are shown. $(L)$ Quantification of results in $K$. Mitotic cells with three or more stretched rDNA lines were scored as having stretched rDNA. Average of two independent experiments \pm SEM. $n=300-596$ cells each. $\left(^{* *}\right) P \leq 0.01$, Student's unpaired $t$-test. $(B, D, F, H, I, K, M)$ DNA was stained with DAPI (blue). Bar, $2 \mu \mathrm{m}$.

centromere side were resolved. Thus, the rDNA repeat arrays constitute a unique domain that (unlike its flanking junctional sequences) remains cohered in mitosis.

To determine the timing of rDNA resolution and its dependence on tankyrase 1, HeLa cells were treated with siRNA (GFP or TNKS1) (immunoblot in Supplemental Fig. S2A) and either arrested in prometaphase (16-h incubation in nocodazole) or released into anaphase $(4.5$-h incubation in nocodazole followed by a $45-\mathrm{min}$ release). FISH analysis of control (GFP siRNA) cells showed rDNA and centromeres unresolved in prometaphase (Fig. 1H, top panels), whereas, upon release into anaphase, centromeres and rDNA resolved into doublets (Fig. 1I, top panels). Analysis of TNKS1 siRNA cells in prometaphase showed a similar pattern as control; rDNA and centromeres were unresolved (Fig. 1H, bottom panels). However, upon release into anaphase, centromeres separated as in control cells, but rDNA did not and instead appeared as stretched lines (Fig. 1I [bottom panels], J). Together, these data indicate that rDNA resolves late after the bulk of the genome in anaphase, dependent on tankyrase 1.

For further analysis, tankyrase siRNA mitotic cells were isolated from asynchronous cultures cells without drug treatment, subjected to FISH analysis, and the images analyzed using deconvolution software. In those cells, the rDNA appeared as thick stretched lines across the metaphase plate (Fig. $1 \mathrm{~K}, \mathrm{~L})$ that stretched between the centromeres (Fig. 1M), similar to the pattern observed in anaphase cells shown above in Figure 1I and consistent with previous studies indicating that tankyrase 1-depleted cells undergo anaphase delay (Dynek and Smith 2004; Kim and Smith 2014). The rDNA stretching was observed in HeLa cells using a probe against the nontranscribed intergenic spacer region (IGS) (rDNA-IGS; see Fig. 1A; Supplemental Fig. S2B,C), confirming that the stretches result from rDNA rather than rRNA. Stretching was observed following treatment with a tankyrase small molecule inhibitor Ti8 in HeLa (Supplemental Fig. S2D,E), BJ (Supplemental Fig. S2F,G), and WI38 (Supplemental Fig. S2H,I) cells, and in HEK293T TNKS1 knockout cells generated by CRISPR/Cas9 (Supplemental Fig. S2J,K; Bhardwaj et al. 2017). Together, these data demonstrate that rDNA stretching occurs upon depletion, inhibition, or deletion of tankyrase 1 in multiple cancer and normal cell types.

The rDNA stretching observed upon tankyrase 1-depletion could reflect a lack of compaction or condensation. We thus determined the role of condensins I and II (Fig. 2A; Ono et al. 2003) in rDNA resolution. Depletion of the common SMC2 subunit in HeLa cells (immunoblot, Fig. 2B) led to rDNA stretching between the centromeres (Fig. 2C,D). To determine whether one or both complexes were required, we depleted complex-specific subunits CAP-D3/condensin II or CAP-D2/condensin I (Fig. 2E, immunoblot). Condensin II localizes to the nucleus during interphase where it participates in the early stages of compaction, whereas condensin I is cytoplasmic and only gains access upon nuclear envelope breakdown in prometaphase (Hirota et al. 2004; Ono et al. 2004). HeLa cell lines stably expressing CAP-D3 or CAP-D2 shRNAs were arrested in nocodazole for $4.5 \mathrm{~h}$, released for $45 \mathrm{~min}$ into anaphase, and analyzed by FISH. As shown in Figure 2, F and G, depletion of CAP-D3, but not CAP$\mathrm{D} 2$, led to rDNA stretching. Immunoprecipitation analysis of endogenous proteins showed that under conditions where the condensin I and II complexes remain assembled (Supplemental Fig. S3), CAP-D3, but not CAP-D2, coimmunoprecipitated with tankyrase 1 (Fig. $2 \mathrm{H}$ ).

Tankyrase binds to its partners though a consensus RXXG(A/P)XG) site. CAP-D3 (but none of the other condensin subunits) has potential sites at positions 5 and 519 (see Fig. 2A). To determine their role in resolution of rDNA, we mutated the essential terminal $\mathrm{G}$ to A to generate single CAP-D3 ${ }^{\mathrm{G} 10 \mathrm{~A}}, \mathrm{CAP}-\mathrm{D} 3^{\mathrm{G} 524 \mathrm{~A}}$, and double CAPD3 ${ }^{\mathrm{GAA}}$ mutants. Vector, wild type, or mutant alleles were introduced into the CAP-D3 shRNA\#2 HeLa cell line and expressed at similar levels (Fig. 2I). FISH analysis 


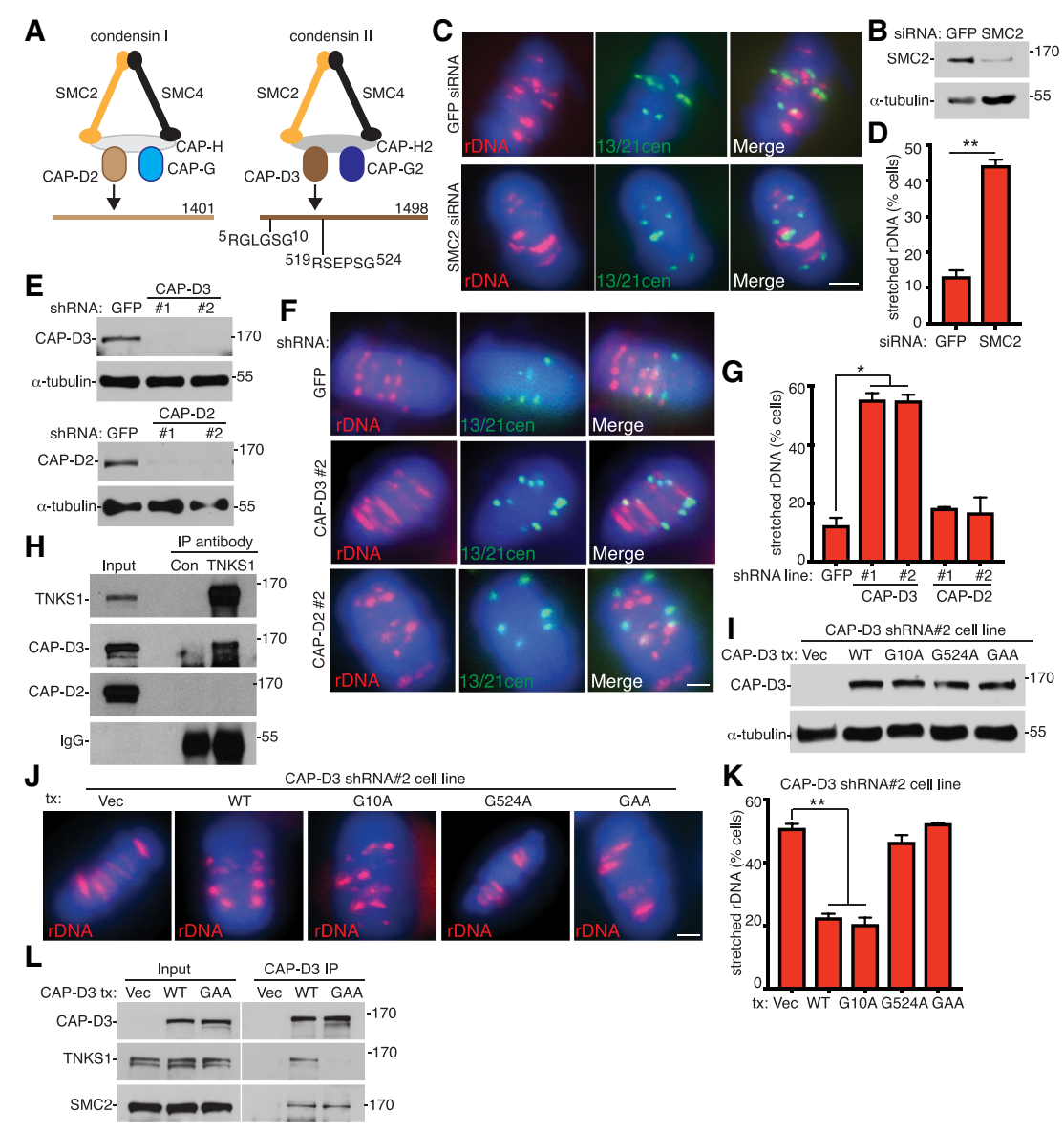

Figure 2. rDNA resolution requires CAP-D3/condensin II. (A) Schematic diagram of condensins I and II. Horizontal lines indicate CAP-D2 and D3. TNKS-binding sites at positions 5 and 519 are indicted in CAP-D3. $(B-D)$ Depletion of SMC2 leads to rDNA stretching. Following a 48-h transfection with GFP or SMC2 siRNA, HeLa cells were analyzed by immunoblot $(B)$ or isolated by mitotic shake-off and analyzed by FISH using rDNA (red) and 13/21 cen (green) probes $(C)$ and quantified $(D)$. Mitotic cells with three or more stretched rDNA lines were scored as having stretched rDNA. Average of two independent experiments $(n=63-70$ cells each $) \pm$ SEM. $\left({ }^{* *}\right) P \leq 0.01$, Student's unpaired $t$-test. $(E-G)$ CAP-D3, not D2, is required for rDNA resolution in anaphase. HeLa cell lines stably expressing CAP-D3 or CAP-D2 shRNAs were analyzed by immunoblot $(E)$ or isolated by mitotic shake-off following a 4.5-h incubation in nocodazole and 45min release into anaphase and analyzed by FISH using rDNA (red) and 13/21 cen (green) probes $(F)$ and quantified $(G)$. Anaphase cells (separated centromeres) with $\geq 3$ stretched rDNA lines were scored as having stretched rDNA. Average of two independent experiments \pm SEM. $n=$ 56-112 cells each. $\left({ }^{*}\right) P \leq 0.05$, Student's unpaired $t$-test. $(H)$ CAP-D3, but not D2, coimmunoprecipitates with TNKS1. Immunoblot analysis of HeLa cells immunoprecipitated with control or TNKS1 antibodies and probed with the indicated antibodies. $(I-K)$ The TNKS-binding site in CAP-D3 at amino acid 519 is required for resolution of cohesion. CAP-D3 shRNA\#2 cells were transfected with GFP-Vec, GFP-CAP-D3 wild type, or GFP-CAP-D3 single G10A or G524, or double GAA mutant plasmids for $18 \mathrm{~h}$ and analyzed by immunoblot $(I)$ or isolated by mitotic shake-off following a $4.5 \mathrm{~h}$ incubation in nocodazole and 45 -min release into anaphase $(J)$ and analyzed by FISH using rDNA (red) probes and quantified $(K)$. Anaphase cells (separated centromeres) with three or more stretched rDNA lines were scored as having stretched rDNA. Average of two independent experiments \pm SEM. $n=56-112$ cells each. $\left({ }^{* *}\right) P \leq 0.01$, Student's unpaired $t$-test. $(L)$ CAP-D3 GAA mutant does not bind tankyrase 1 but retains its association with SMC2. Immunoblot analysis of U2OS cells immunoprecipitated with anti-GFP antibody following transfection with GFP-Vec, GFP-CAP-D3 wild type, or GFP-CAP-D3 GAA and probed with the indicated antibodies. $(C, F, J)$ DNA was stained with DAPI (blue). Bar, $2 \mu \mathrm{m}$.

of anaphase cells showed that rDNA stretching was rescued by introduction of wild-type CAP-D3 (Fig. 2J,K). Rescue was also observed with CAP-D3 ${ }^{\mathrm{G} 10 \mathrm{~A}}$, indicating that the amino terminal tankyrase binding site was not critical (Fig. 2J,K). In contrast, the CAP-D $3^{\mathrm{G} 524 \mathrm{~A}}$ mutant and the CAP-D3 ${ }^{\mathrm{GAA}}$ double mutant did not rescue rDNA stretching (Fig. 2J,K), indicating a requirement for the second (position 519) tankyrase binding site. Immunoprecipitation analysis showed that in contrast to wild-type CAP-D3, CAP$\mathrm{D} 3^{\mathrm{GAA}}$ did not coimmunoprecipitate tankyrase 1, but, importantly, retained its association with SMC2/condensin (Fig. 2L). Together, these data indicate that tankyrase 1 binds CAP-D3, and binding is required for resolution of rDNA. The rDNA stretching that we observed in anaphase is specific to the rDNA, as we did not observe stretching with the PJ or DJ probes in tankyrase 1- or CAP-D3-depleted anaphase cells (Supplemental Fig. S4).

Condensin action is a prerequisite for topo II-mediated decatenation in budding yeast $\left(\mathrm{D}^{\prime}\right.$ Ambrosio et al. 2008; Baxter et al. 2011; Charbin et al. 2014). To determine the role of topo IIa in rDNA resolution in human cells, HeLa cells were treated with the topo IIa inhibitors etoposide or ICRF193 for $2 \mathrm{~h}$, isolated by mitotic shakeoff, and analyzed by rDNA FISH. As shown in Figure 3, A and B, inhibition of topo Ila led to rDNA stretching. We also observed some centromere stretching; however, it occurred at a much lower frequency than rDNA stretching and only at a high concentration of etoposide or with ICRF-193 (Supplemental Fig. S5). The similarity of the rDNA phenotypes raised the possibility that the rDNA stretches observed in CAP-D3- and tankyrase 1-depleted cells resulted from topo IIa inaction. To determine whether overexpression of topo IIa could rescue the rDNA stretches induced by CAPD3 or tankyrase 1 depletion, plasmids expressing topo IIa, tankyrase 1, CAP-D3, or a vector control were introduced into HeLa cells stably expressing TNKS1 shRNA (Supplemental Fig. S6A, immunoblot) or CAPD3 shRNA\#2 (see immunoblot, Fig. 2E). Expression of the transfected proteins was confirmed by immunoblot analysis (Supplemental Fig. S6B,C). In TNKS1-depleted cells, topo IIa rescued the rDNA stretches to a similar extent as TNKS1 (Fig. 3C,D). In contrast, CAP-D3 did not rescue and was similar to the vector. In CAP-D3-depleted cells, topo IIa rescued the rDNA stretches, as did CAP-D3 (Fig. $3 \mathrm{E}, \mathrm{F}$. In contrast, TNKS1 did not rescue and was similar to the vector. Together, these data indicate that topo Ila overexpression can compensate for the absence of CAP-D3 or TNKS1. Additionally, the observation that CAP-D3 overexpression does not compensate for TNKS1-depletion and vice versa indicate that they are both required for rDNA resolution. 
A

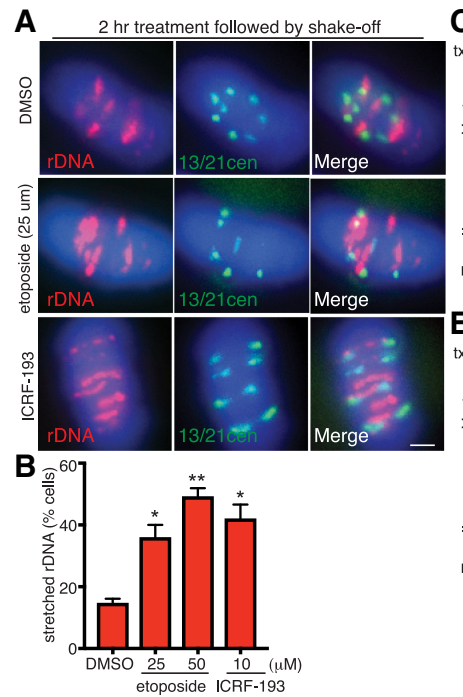

C
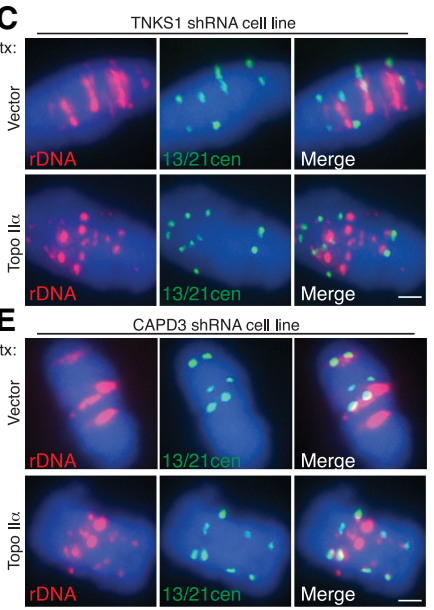

D TNKS1 ShRNA cell line

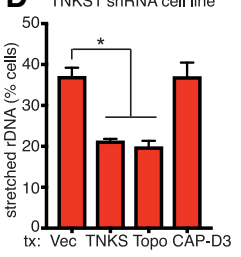

F CAPD3 shRNA cell line

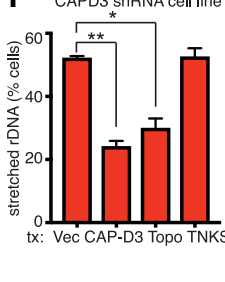

$\mathbf{G}$
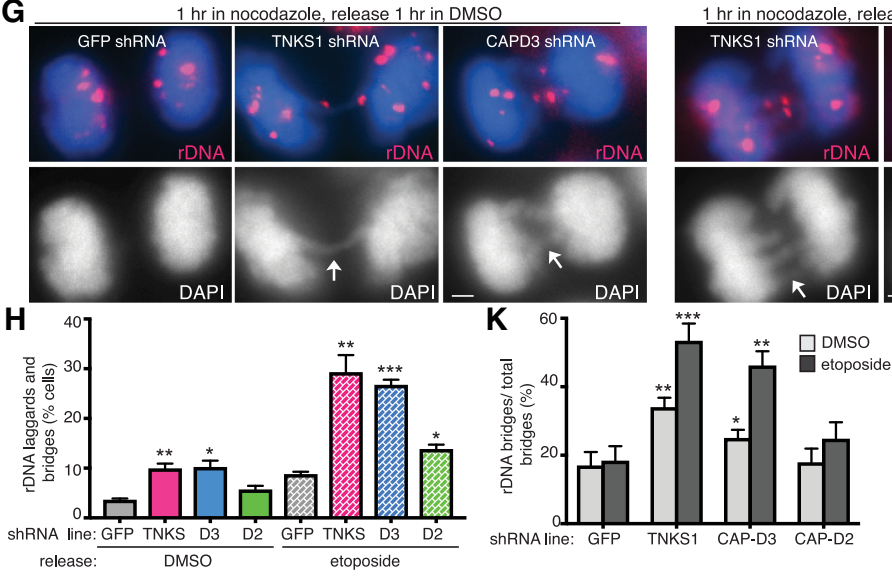

I
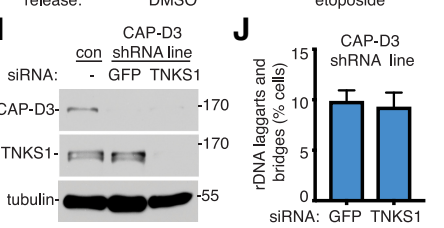

Figure 3. Topo II a is required for rDNA resolution. $(A, B)$ Inhibition of Topo IIa leads to rDNA stretching. HeLa cells were isolated by mitotic shake-off following a 2 -h treatment with (vehicle) DMSO or the Topo II $a$ inhibitors etoposide or ICRF-193 and analyzed by FISH using rDNA (red) and $13 / 21$ cen (green) probes $(A)$ and quantified $(B)$. Mitotic cells with three or more stretched rDNA lines were scored as having stretched rDNA. Average of two independent experiments $(n=50-95$ cells each $) \pm$ SEM. $\left(^{*}\right) P \leq 0.05,(* *) P \leq 0.01$, Student's unpaired $t$-test. $(C-F)$ Topo IIa overexpression rescues stretched rDNA in TNKS1- or CAP-D3-depleted cells. TNKS1 $(C, D)$ or CAP-D3 $(E, F)$ shRNA HeLa cell lines were transfected with vector, Flag-TNKS1, Flag-Topo IIa, or GFP-CAP-D3 plasmids for $18 \mathrm{~h}$; incubated for an additional $4.5 \mathrm{~h}$ in nocodazole; released for 45 min into anaphase; isolated by mitotic shake-off; and analyzed by FISH using rDNA (red) and $13 / 21$ cen (green) probes $(C, E)$ and quantified $(D, F)$. Anaphase cells (separated centromeres) with three or more stretched rDNA lines were scored as having stretched rDNA. Average of two independent experiments \pm SEM. $(D) n=51-111$ cells each. $(F) n=76-127$ cells each. $\left({ }^{*}\right) P \leq$ $0.05,\left({ }^{*}\right) P \leq 0.01$, Student's unpaired $t$-test. $(G-K)$ TNKS1 or CAP-D3 depletion leads to rDNA segregation defects that are exacerbated by topo Ila inhibition. HeLa cell lines stably expressing GFP, TNKS1, CAP-D3, or CAP-D2 shRNA were treated with nocodazole for $1 \mathrm{~h}$ and released in DMSO or $50 \mu \mathrm{m}$ etoposide for $1 \mathrm{~h}$ and analyzed by FISH using an rDNA probe (red) $(G)$ and quantified $(H)$. Arrows indicate DAPI-staining bridges. Late anaphase cells with rDNA laggards and bridges were scored. Average of three independent experiments \pm SEM. $n=45-112$ cells each. $\left({ }^{*}\right)$ $\left.P \leq 0.05 ;\left({ }^{* *}\right) P \leq 0.01 ;{ }^{* * *}\right) P \leq 0.001$, Student's unpaired $t$-test. $(I, J)$ Double depletion of TNKS1 and CAP-D3 does not exacerbate the phenotype. (I) CAP-D3 shRNA cells treated with GFP or TNKS1 siRNA for $48 \mathrm{~h}$ were analyzed by immunoblot $(I)$ or FISH $(J)$ following treatment with nocodazole for $1 \mathrm{~h}$ and release in DMSO for $1 \mathrm{~h}$ and quantified. Late anaphase cells with rDNA laggards and bridges were scored. Average of three independent experiments \pm SEM. $n=41-58$ cells each. $(K)$ Graphical representation of the frequency of rDNA-positive DAPI bridges versus total DAPI bridges from $H .(A, C, E, G)$ DNA was stained with DAPI (blue). Bar, $2 \mu \mathrm{m}$.
To determine whether impaired rDNA resolution (indicated by rDNA stretching) led to rDNA segregation defects (lagging rDNA and bridges) as mitosis progressed to late anaphase, HeLa shRNA cell lines (GFP, TNKS1, CAP-D3, and CAP-D2) were arrested in prometaphase by a brief (1-h) treatment with nocodazole, released into anaphase/telophase for $1 \mathrm{~h}$, isolated by mitotic shake-off, and analyzed by FISH. As shown in Figure 3, G/three left panels) and $\mathrm{H}$, depletion of TNKS1 or CAP-D3 (but not CAP-D2) resulted in a threefold increase in rDNA segregation defects compared with the GFP shRNA control. Notably, depletion of TNKS1 in CAP-D3-depleted cells (confirmed by immunoblot in Fig. 3I) did not exacerbate the phenotype (Fig. $3 \mathrm{~J}$ ), indicating that TNKS1 and CAP-D3 act in the same pathway to promote resolution and segregation of rDNA.

To determine whether TNKS1 and CAP-D3 are required to facilitate topo IIa-mediated decatenation of rDNA, we performed the analysis as described above, but included the topo Ila inhibitor etoposide in the 1-h release from nocodazole. As shown in Figure 3, G (two right panels) and $\mathrm{H}$, inhibition of topo Ila led to an increase in rDNA segregation defects that was most prominent in the TNKS1- and CAP-D3depleted cells. CAP-D2-depleted cells showed a weaker, but significant, increase in rDNA segregation defects, suggesting that CAP-D2/condensin I may be important for topo IIa-mediated decatenation of rDNA late in mitosis. Strikingly, calculation of the frequency of rDNA bridges relative to total DAPI-staining bridges showed a significant increase in TNKS1- and CAP-D3-depleted cells (compared with GFP or CAP-D2) that was exacerbated in the presence of etoposide; up to $53 \%$ of the DAPI-staining DNA bridges contained rDNA (Fig. 3K). Together, these data indicate the tankyrase 1 and CAP-D3 facilitate topo IIamediated segregation of rDNA.

We next determined the impact of rDNA segregation defects on genome stability in tankyrase 1-depleted cells without drug treatment. As shown in Figure 4A,B, TNKS1 siRNA-treated HeLa cells isolated by mitotic shakeoff from asynchronous cultures showed DAPI-staining DNA bridges and lagging rDNA in late stages of mitosis. Such unresolved rDNA could lead to nondisjunction. Indeed, FISH analysis showed a significant increase in aneuploidy for the rDNA- 


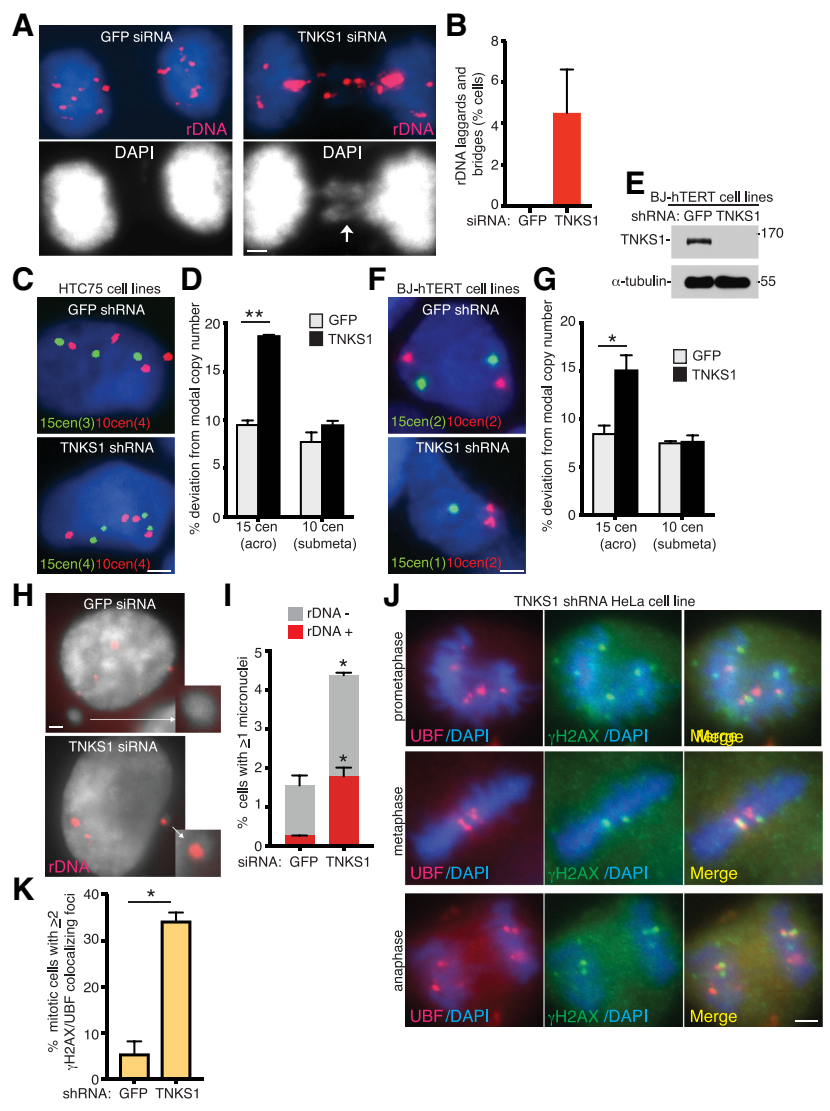

Figure 4. Impaired rDNA segregation leads to genomic instability. $(A, B)$ TNKS1-depleted cells have rDNA bridges in late anaphase. HeLa cells were isolated by mitotic shake-off from asynchronously growing cultures following 48-h transfection with GFP or TNKS1 siRNA and analyzed by FISH using an rDNA probe (red; deconvolved images are shown $)(A)$ and quantified $(B)$. Arrow indicates DAPI-staining bridge. Late anaphase cells with rDNA laggards and bridges were scored. Average of two independent experiments \pm SEM. $n=25-34$ late anaphase cells each. $(C-G)$ Increased aneuploidy of the acrocentric chromosome 15 in TNKS1-depleted cells. FISH analysis of interphase cells from $(C)$ HTC75 or $(F)$ BJ-hTERT cells stably expressing GFP or TNKS1 shRNA using a 15 cen (green) or 10 cen (red) probe. $(D, G)$ Quantification of the deviation from the modal copy number for $C$ and $F$, respectively. $(D)$ Average of two independent experiments \pm SEM. $n=201-222$ cells each. ( $G$ ) Average of three independent experiments \pm SEM. $n=108-140$ cells each. $\left({ }^{*}\right) P \leq 0.05 ;(* *) P \leq 0.01$, Student's unpaired $t$-test. $(E)$ Immunoblot analysis of BJ-hTERT cell lines stably expressing GFP or TNKS1 shRNA. $(H, I)$ Increased rDNA-containing micronuclei in TNKS1-depleted cells. FISH analysis of HeLa cells following 72-h transfection with GFP or TNKS1 siRNA with an rDNA probe (red). (I) Quantification of interphase cells containing one or more micronuclei without $(-)$ or with $(+)$ rDNA. Average of two independent experiments \pm SEM. $n=301-388$ cells each. $\left({ }^{*}\right) P \leq 0.05$, Student's unpaired $t$-test. $(J, K)$ Increased rDNA associated DNA damage foci in TNKS1-depleted cells. $(J)$ Asynchronous HeLa cells were fixed in $3.7 \%$ paraformaldehyde and analyzed by immunofluorescence by dual staining with anti-UBF (red) and $\gamma \mathrm{H} 2 \mathrm{AX}$ (green) antibodies. $(K)$ Quantification of mitotic cells containing two or more colocalizing foci. Average of two independent experiments \pm SEM. $n=36-65$ cells each. $\left({ }^{*}\right) P \leq 0.05$, Student's unpaired $t$-test.

containing acrocentric chromosome 15 compared with a control nonacrocentric chromosome 10 in TNKS1 shRNA-depleted HTC75 cells (Fig. 4C,D) and in TNKS1 shRNA-depleted BJ TERT-immortalized fibroblasts (Fig. 4E-G). We observed a similar increase in aneuploidy for chromosome 15 compared with two other nonacrocentric chromosomes 6 and 16 (Supplemental Fig. S7). In addition to nondisjunction, rDNA segregation defects could lead to micronuclei formation. TNKS1 siRNA-depleted HeLa cells exhibited a threefold increase in micronuclei compared with control cells, with a sevenfold increase in micronuclei that were rDNA-positive (Fig. 4H,I). Finally, we queried the consequence of rDNA segregation defects on the induction of rDNA-specific DNA damage. Asynchronously growing TNKS1 shRNA or GFP shRNA control HeLa cells were subjected to dual immunofluorescence analysis using antibodies against the DNA damage marker $\gamma \mathrm{H} 2 \mathrm{AX}$ and UBF for the rDNA. As shown in Figure 4J,K, 34\% of TNKS1-depleted mitotic cells (at all stages of mitosis) showed two or more colocalizing foci compared with $6 \%$ in control, indicating profound consequences for genomic stability at rDNA.

Our studies show that resolution of human rDNA depends on tankyrase 1, condensin II, and topo IIa. We propose that tankyrase 1 acts together with condensin II by direct interaction with the CAP-D3 subunit to promote topo II-mediated decatenation of rDNA. We showed that resolution of rDNA occurs in anaphase after the bulk of the genome. Previous studies in human cells have shown that resolution of sister chromatid arms is complete by the end of prophase, dependent on condensin II and topo IIa (Abe et al. 2011; Nagasaka et al. 2016) and that conden$\sin$ II localizes to the nucleus in interphase and acts as early as S phase to initiate resolution (Ono et al. 2013). However, despite its nuclear localization, condensin II appears to be excluded from nucleoli and thus may not gain access to the rDNA (Ono et al. 2013). Exposure to condensin II may occur only after the rDNA is released from the nucleolus upon nuclear envelope breakdown in late prophase. This window would coincide with repression of rDNA transcription in human cells (Prescott 1964), which (at least in budding yeast) is required for condensin binding (Tomson et al. 2006; Clemente-Blanco et al. 2009). It is tempting to speculate that resolution of rDNA is delayed in humans, as in yeast, to allow repression of transcription as a prerequisite for condensin binding.

The importance of timely resolution of the rDNA is illustrated by the impact of aberrant segregation on genome integrity, reflected by the increase in rDNA bridges, rDNA-containing micronuclei, aneuploidy of the rDNAcontaining chromosome 15 , and rDNA-associated DNA damage in mitosis. Interestingly, while rDNA stretching was greatly stimulated by depletion of condensin II or tankyrase 1, or inhibition of topo II a, we observed some level of stretching in untreated control cells (ranging from $4 \%$ in normal cells up to $20 \%$ in cancer cells), suggesting that delayed resolution of rDNA could pose a heretofore unappreciated threat to genome integrity.

\section{Materials and methods}

\section{Chromosome-specific FISH}

Mitotic cells were isolated by shake-off, fixed, and processed as described previously (Dynek and Smith 2004) and hybridized with green centromere probes (15 cen and 13/21 cen) and red AcroP (rDNA) probes (Cytocell) or with green DJ or PJ probes and red rDNA-IGS probes (van Sluis et al. 2016).

\section{Image analysis}

Images were acquired using a microscope (Axioplan 2; Carl Zeiss, Inc.) with a Plan Apochrome 63x NA 1.4 oil immersion lens (Carl Zeiss, Inc.) 
and a digital camera (Hamamatsu Photonics, C4742-95). Images were acquired and processed using Openlab software (Perkin Elmer).

\section{Statistical analysis}

Statistical analysis was performed using Prism 7 software. Student's unpaired $t$-test was applied. Data are shown as mean \pm SEM (standard error of the mean); $P<0.05$ values were considered significant. A detailed description of the Materials and Methods is in the Supplemental Material.

\section{Acknowledgments}

We thank Smith laboratory members and Tom Meier for critical reading of the manuscript, and Christine Farr and Toru Hirota for providing plasmids. Research reported in this publication was supported by the National Institutes of Health under award number R01GM129780.

Author contributions: S.S., Z.D., and K.B. conceived the project and designed the experiments. Z.D. and K.B. performed the experiments. B.M. provided essential materials. S.S., Z.D., K.B., and B.M. analyzed and interpreted the data. S.S. and Z.D. wrote the first draft. S.S., Z.D., K.B., and B.M. wrote the final manuscript.

\section{References}

Abe S, Nagasaka K, Hirayama Y, Kozuka-Hata H, Oyama M, Aoyagi Y, Obuse C, Hirota T. 2011. The initial phase of chromosome condensation requires Cdk1-mediated phosphorylation of the CAP-D3 subunit of condensin II. Genes Dev 25: 863-874. doi:10.1101/gad.2016411

Baxter J, Sen N, Martinez VL, De Carandini ME, Schvartzman JB, Diffley JFX, Aragon L. 2011. Positive supercoiling of mitotic DNA drives decatenation by topoisomerase II in eukaryotes. Science 331: 1328-1332. doi:10.1126/science.1201538

Bhardwaj A, Yang Y, Ueberheide B, Smith S. 2017. Whole proteome analysis of human tankyrase knockout cells reveals targets of tankyrasemediated degradation. Nature Commun 8: 2214. doi:10.1038/s41467017-02363-w

Bisht KK, Daniloski Z, Smith S. 2013. SA1 binds directly to DNA through its unique AT-hook to promote sister chromatid cohesion at telomeres. J Cell Sci 126: 3493-3503. doi:10.1242/jcs.130872

Canudas S, Smith S. 2009. Differential regulation of telomere and centromere cohesion by the Scc3 homologues SA1 and SA2, respectively, in human cells. J Cell Biol 187: 165-173. doi:10.1083/jcb.200903096

Charbin A, Bouchoux C, Uhlmann F. 2014. Condensin aids sister chromatid decatenation by topoisomerase II. Nucleic Acids Res 42: 340-348. doi:10.1093/nar/gkt882

Clemente-Blanco A, Mayan-Santos M, Schneider DA, Machin F, Jarmuz A, Tschochner H, Aragon L. 2009. Cdcl4 inhibits transcription by RNA polymerase I during anaphase. Nature 458: 219-222. doi:10.1038/nature07652

D'Ambrosio C, Kelly G, Shirahige K, Uhlmann F. 2008. Condensin-dependent rDNA decatenation introduces a temporal pattern to chromosome segregation. Curr Biol 18: 1084-1089. doi:10.1016/j.cub.2008.06.058

D'Amours D, Stegmeier F, Amon A. 2004. Cdc14 and condensin control the dissolution of cohesin-independent chromosome linkages at repeated DNA. Cell 117: 455-469. doi:10.1016/S0092-8674(04)00413-1

Dynek JN, Smith S. 2004. Resolution of sister telomere association is required for progression through mitosis. Science 304: 97-100. doi:10 $.1126 /$ science. 1094754

Floutsakou I, Agrawal S, Nguyen TT, Seoighe C, Ganley ARD, McStay B. 2013. The shared genomic architecture of human nucleolar organizer regions. Genome Res 23: 2003-2012. doi:10.1101/gr.157941.113

Gonzalez IL, Sylvester JE. 1995. Complete sequence of the 43-kb human ribosomal DNA repeat: analysis of the intergenic spacer. Genomics 27: 320-328. doi:10.1006/geno.1995.1049

Guacci V, Koshland D, Strunnikov A. 1997. A direct link between sister chromatid cohesion and chromosome condensation revealed through the analysis of MCD1 in S. cerevisiae. Cell 91: 47-57. doi:10.1016/ S0092-8674(01)80008-8

Henderson AS, Warburton D, Atwood KC. 1972. Location of ribosomal DNA in the human chromosome complement. Proc Natl Acad Sci 69: 3394-3398. doi:10.1073/pnas.69.11.3394
Hirota T, Gerlich D, Koch B, Ellenberg J, Peters JM. 2004. Distinct functions of condensin I and II in mitotic chromosome assembly. J Cell Sci 117: 6435-6445. doi:10.1242/jcs.01604

Kim MK, Smith S. 2014. Persistent telomere cohesion triggers a prolonged anaphase. Mol Biol Cell 25: 30-40. doi:10.1091/mbc.e13-08-0479

Kschonsak M, Haering CH. 2015. Shaping mitotic chromosomes: from classical concepts to molecular mechanisms. Bioessays 37: 755-766. doi:10.1002/bies.201500020

McStay B. 2016. Nucleolar organizer regions: genomic 'dark matter' requiring illumination. Genes Dev 30: 1598-1610. doi:10.1101/gad .283838 .116

Michaelis C, Ciosk R, Nasmyth K. 1997. Cohesins: chromosomal proteins that prevent premature separation of sister chromatids. Cell 91:35-45. doi:10.1016/S0092-8674(01)80007-6

Nagasaka K, Hossain MJ, Roberti MJ, Ellenberg J, Hirota T. 2016. Sister chromatid resolution is an intrinsic part of chromosome organization in prophase. Nat Cell Biol 18: 692-699. doi:10.1038/ncb3353

Nemeth A, Grummt I. 2018. Dynamic regulation of nucleolar architecture. Curr Opin Cell Biol 52: 105-111. doi:10.1016/j.ceb.2018.02.013

Ofir R, Yalon-Hacohen M, Segev Y, Schultz A, Skorecki KL, Selig S. 2002. Replication and/or separation of some human telomeres is delayed beyond S-phase in pre-senescent cells. Chromosoma 111: 147-155. doi:10.1007/s00412-002-0199-z

Ono T, Losada A, Hirano M, Myers MP, Neuwald AF, Hirano T. 2003. Differential contributions of condensin I and condensin II to mitotic chromosome architecture in vertebrate cells. Cell 115: 109-121. doi:10 $.1016 /$ S0092-8674(03)00724-4

Ono T, Fang Y, Spector DL, Hirano T. 2004. Spatial and temporal regulation of condensins I and II in mitotic chromosome assembly in human cells. Mol Biol Cell 15: 3296-3308. doi:10.1091/mbc.e04-03-0242

Ono T, Yamashita D, Hirano T. 2013. Condensin II initiates sister chromatid resolution during S phase. J Cell Biol 200: 429-441. doi:10.1083/jcb 201208008

Prescott DM. 1964. Cellular sites of RNA synthesis. Prog Nucleic Acid Res Mol Biol 3: 33-57. doi:10.1016/S0079-6603(08)60738-2

Shintomi K, Hirano T. 2010. Sister chromatid resolution: a cohesin releasing network and beyond. Chromosoma 119: 459-467. doi:10.1007/ s00412-010-0271-z

Smith S, Giriat I, Schmitt A, de Lange T. 1998. Tankyrase, a poly(ADP-ribose) polymerase at human telomeres. Science 282: 1484-1487. doi:10 $.1126 /$ science.282.5393.1484

Stults DM, Killen MW, Pierce HH, Pierce AJ. 2008. Genomic architecture and inheritance of human ribosomal RNA gene clusters. Genome Res 18: 13-18. doi:10.1101/gr.6858507

Sullivan M, Higuchi T, Katis VL, Uhlmann F. 2004. Cdc14 phosphatase induces rDNA condensation and resolves cohesin-independent cohesion during budding yeast anaphase. Cell 117: 471-482. doi:10.1016/S00928674(04)00415-5

Tomson BN, D'Amours D, Adamson BS, Aragon L, Amon A. 2006. Ribosomal DNA transcription-dependent processes interfere with chromosome segregation. Mol Cell Biol 26: 6239-6247. doi:10.1128/MCB $.00693-06$

Torres-Rosell J, Machin F, Jarmuz A, Aragon L. 2004. Nucleolar segregation lags behind the rest of the genome and requires Cdc14p activation by the FEAR network. Cell Cycle 3: 496-502. doi:10.4161/cc.3.4.802

Uhlmann F, Lottspeich F, Nasmyth K. 1999. Sister-chromatid separation at anaphase onset is promoted by cleavage of the cohesin subunit Scc1. Nature 400: 37-42. doi:10.1038/21831

Uhlmann F, Wernic D, Poupart MA, Koonin EV, Nasmyth K. 2000. Cleavage of cohesin by the $\mathrm{CD}$ clan protease separin triggers anaphase in yeast. Cell 103: 375-386. doi:10.1016/S0092-8674(00)00130-6

van Sluis M, van Vuuren C, McStay B. 2016. The relationship between human nucleolar organizer regions and nucleoli, probed by 3D-ImmunoFISH. Methods Mol Biol 1455: 3-14. doi:10.1007/978-1-4939-3792-9_1

Waizenegger IC, Hauf S, Meinke A, Peters JM. 2000. Two distinct pathways remove mammalian cohesin from chromosome arms in prophase and from centromeres in anaphase. Cell 103: 399-410. doi:10.1016/ S0092-8674(00)|00132-X

Wang BD, Yong-Gonzalez V, Strunnikov AV. 2004. Cdc14p/FEAR pathway controls segregation of nucleolus in $S$. cerevisiae by facilitating condensin targeting to rDNA chromatin in anaphase. Cell Cycle 3: 960-967. 


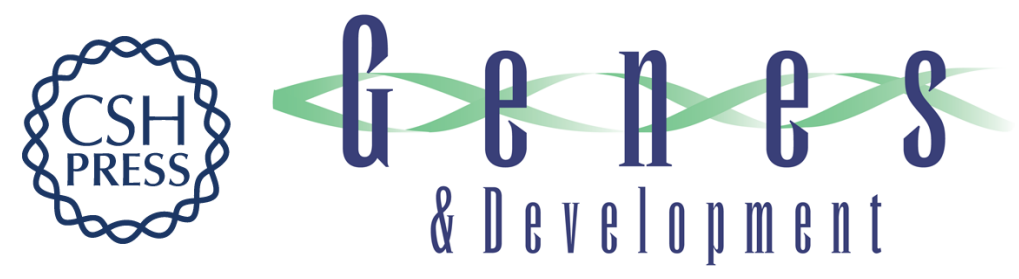

\section{Resolution of human ribosomal DNA occurs in anaphase, dependent on tankyrase 1, condensin II, and topoisomerase II $\alpha$}

Zharko Daniloski, Kamlesh K. Bisht, Brian McStay, et al.

Genes Dev. 2019, 33: originally published online February 25, 2019

Access the most recent version at doi:10.1101/gad.321836.118

\section{Supplemental http://genesdev.cshlp.org/content/suppl/2019/02/23/gad.321836.118.DC1 \\ Material}

References This article cites 37 articles, 15 of which can be accessed free at:

http://genesdev.cshlp.org/content/33/5-6/276.full.html\#ref-list-1

Creative This article is distributed exclusively by Cold Spring Harbor Laboratory Press for the first

Commons six months after the full-issue publication date (see

License http://genesdev.cshlp.org/site/misc/terms.xhtml). After six months, it is available under a Creative Commons License (Attribution-NonCommercial 4.0 International), as described at http://creativecommons.org/licenses/by-nc/4.0/.

Email Alerting Receive free email alerts when new articles cite this article - sign up in the box at the top Service right corner of the article or click here.

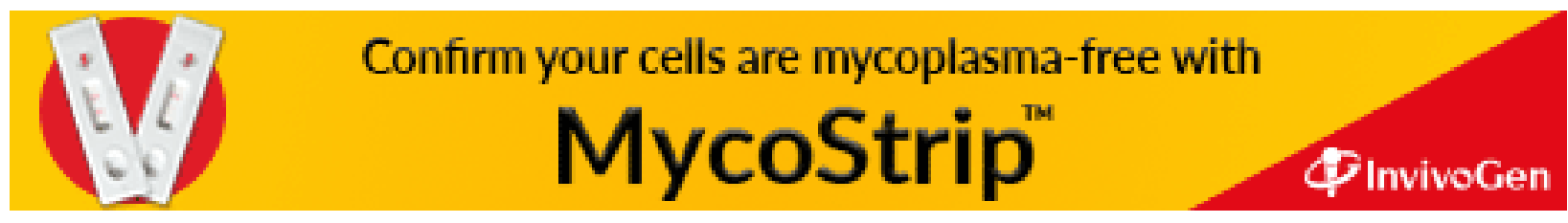

